\title{
Effect of a quality-controlled fermented nutraceutical on skin aging markers: An antioxidant-control, double-blind study
}

\author{
GIUSEPPE BERTUCCELLI ${ }^{1}$, NICOLA ZERBINATI ${ }^{2}$, MASSIMILIANO MARCELLINO $^{1}$, \\ NAVALPUR SHANMUGAM NANDA KUMAR ${ }^{3}$, FANG HE ${ }^{4}$, VLADIMIR TSEPAKOLENKO ${ }^{5}$, \\ JOSEPH CERVI ${ }^{1}$, ALDO LORENZETTI ${ }^{1}$ and FRANCESCO MAROTTA ${ }^{1}$ \\ ${ }^{1}$ ReGenera Research Group for Aging Intervention, Milan 20154; ${ }^{2}$ Dermatology Unit, CMP-Medical Center and Laboratories, \\ Pavia 27100, Italy; ${ }^{3}$ Department of Pediatrics, Mucosal Immunology and Biology Research Center, \\ Massachusetts General Hospital and Harvard Medical School, Boston, MA 02114, USA; \\ ${ }^{4}$ Department of Nutrition and Food Hygiene, West China School of Public Health, \\ Sichuan University, Chengdu, Sichuan 610065, P.R. China; ${ }^{5}$ Virtus Medical Center, \\ Ukraine Institute of Plastic Surgery and Dermatology, Odessa 68355, Ukraine
}

Received March 30, 2015; Accepted September 28, 2015

DOI: $10.3892 /$ etm.2016.3011

\begin{abstract}
The aim of the present study was to determine whether oral supplementation with a fermented papaya preparation (FPP-treated group) or an antioxidant cocktail (antioxidant-control group, composed of $10 \mathrm{mg}$ trans-resveratrol, $60 \mu \mathrm{g}$ selenium, $10 \mathrm{mg}$ vitamin $\mathrm{E}$ and $50 \mathrm{mg}$ vitamin $\mathrm{C}$ ) was able to improve the skin antioxidant capacity and the expression of key skin genes, while promoting skin antiaging effects. The study enrolled 60 healthy non-smoker males and females aged 40-65 years, all of whom showed clinical signs of skin aging. The subjects were randomly divided into two matched groups, and were administered FPP or antioxidant treatment of a $4.5 \mathrm{~g}$ /day sachet sublingually twice a day for 90 days in a double-blind fashion. The parameters investigated were: Skin surface, brown spots, skin evenness, skin moisturization, elasticity (face), redox balance, nitric oxide (NO) concentration, and the expression levels of key genes (outer forearm sample). As compared with the baseline (day 0) and antioxidant-control values, FPP-treated subjects showed a significant improvement in skin evenness, moisturization and elasticity. The two treatments improved the MDA and SOD skin concentrations, but only the FPP-treated group showed a higher SOD level and a significant NO increase, along with significant upregulation of acquaporin-3 and downregulation of the potentially pro-aging/carcinogenetic cyclophilin-A and CD147 genes $(\mathrm{P}<0.05)$. Progerin was unaffected in both treatment groups.
\end{abstract}

Correspondence to: Professor Francesco Marotta, ReGenera Research Group for Aging-Intervention, 12 Piazza Firenze, Milan 20154, Italy

E-mail: fmarchimede@libero.it

Key words: fermented papaya preparation, skin aging, photoaging, gene expression, skin elasticity, skin moisturization, antioxidant defence
In conclusion, these findings suggest that orally-administered FPP showed a consistent biological and gene-regulatory improvement in the skin, as was also demonstrated in previous experimental and clinical trials testing other tissues, while common oral antioxidants had only a minor effect.

\section{Introduction}

Aging is associated with a wide range of impairments of the regulatory systems, particularly systems that are associated with surveillance and defence mechanisms (1). The skin, as the body's largest organ, follows an oxidant/antioxidant defense path and abnormalities leading to photoaging, chronoaging and inflammation are mediated by reactive oxygen species (ROS) (2). The true biological impact of the direct topical administration of a wide variety of natural and synthetic compounds on skin health remains unclear (3). Considering that the hydrophobic nature of the upper layer of the skin is the stratum corneum, specific oral phytonutrients have been proven to improve certain clinical aspects of skin health (4-6). Although the skin possesses a multifaceted antioxidant defense structure, unbalanced and enduring exposure to ultraviolet (UV) radiation can overwhelm the dermal antioxidant capability, resulting in oxidative damage that may lead to skin immune system dysregulation, premature aging and development of skin cancer (7). Chronic sun exposure results in photoaged skin (8), characterized by the premature occurrence of aging signs on the skin, which displays distinct morphological changes of the epidermal and dermal compartments, the pigmentary system and the vasculature $(9,10)$. These changes are also accompanied by an increased skin cancer occurrence (11). Furthermore, changes of intrinsic aging have to be considered, and these are typically observed in chronically sun-protected areas where aging is mainly attributed to intrinsic factors, including genetics and changes in the endocrine environment, reflecting the degradation processes of the entire organism (12). Previous in vitro data obtained from human epithelial skin cells have shown that studies on this type 
of tissue may provide useful information when investigating the same embryologic-derived tissues, such as tissues from the central nervous system (13). More recently, a study has reported that a resveratrol-procyanidin-based nutraceutical was able to exert a beneficial skin antiaging effect (14). However, the study only partially addressed skin clinicobiochemical evaluation and did not assess gene expression.

A number of genes and associated proteins have recently emerged as relevant for skin health (15). Among them, aquaporin-3 (AQP-3) is expressed in keratinocytes and fibroblasts, and regulates water movement across the plasma membrane via diffusion through the lipid bilayer, as well as being involved in wound healing (16). The common upregulation of AQP-3 by antioxidants has been suggested to beneficially modulate this protein in order to maintain skin health, cell turnover and skin hydration (15). Cyclophilin A (CyPA), a member of the immunophilin family, has been recently found to play an important role in impairing skin DNA repair mechanisms (17), while also affecting systemic oxidative stress-inflammatory response $(18,19)$. The transmembrane glycoprotein CD147, a cell surface receptor of CyPA that belongs to the immunoglobulin superfamily, plays a relevant role in CyPA-mediated signal transmission and chemotactic activity (20). Finally, a recent study has suggested that UVA may induce progerin mRNA and protein expression in dermal fibroblasts as a novel mechanism of UV-accelerated skin aging (21).

A fermented papaya preparation (FPP) has been shown to possess effective antioxidant properties in previous in vitro and in vivo studies (22-24). Therefore, the aim of the current study was to determine whether nutraceutical treatment with a supplement composed of GMP- and ISO9001-certified FPP was able to improve the skin antioxidant capacity and the expression of key skin genes, while promoting clinically evident skin antiaging effects.

\section{Materials and methods}

Ethics. All procedures were approved by an independent Ethical Committee for non-pharmacological research (ReGenera Research Group for Aging Intervention, Milan, Italy). Each subject recruited for the study was fully informed and treated in compliance with the guidelines of the Declaration of Helsinki. All skin samples were obtained under the written informed consent of the donors.

FPP. The FPP used in the present study was obtained from Carica papaya L. cultivated in Hawaii, following yeast fermenting for 10 months and batch-to-batch checking at the Osato Research Institute (Gifu, Japan). The final composition of FPP per $100 \mathrm{~g}$ is as follows: $90.7 \mathrm{~g}$ carbohydrates, $17 \mu \mathrm{g}$ vitamin B6, $2 \mu \mathrm{g}$ folic acid, $2.5 \mathrm{mg}$ calcium, $16.9 \mathrm{mg}$ potassium, $240 \mu \mathrm{g}$ niacin, $4.6 \mathrm{mg}$ magnesium, $14 \mu \mathrm{g}$ copper, $75 \mu \mathrm{g}$ zinc, $16 \mathrm{mg}$ arginine, $6 \mathrm{mg}$ lysine, $5 \mathrm{mg}$ hystidine, $11 \mathrm{mg}$ phenylalanine, $9 \mathrm{mg}$ tyrosine, $18 \mathrm{mg}$ leucine, $9 \mathrm{mg}$ isoleucine, $5 \mathrm{mg}$ methionine, $13 \mathrm{mg}$ valine, $11 \mathrm{mg}$ glycine, $8 \mathrm{mg}$ proline, $37 \mathrm{mg}$ gluthamic acid, $11 \mathrm{mg}$ serine, $8 \mathrm{mg}$ treonine, $27 \mathrm{mg}$ aspartic acid, and $2 \mathrm{mg}$ triptophane.

Subjects and study design. The study enrolled 60 healthy non-smoker males and females with an age of 40-65 years, all of whom showed clinical signs of skin aging (including wrinkles, dull complexion and brown spots). The inclusion criteria were as follows: Caucasian ethnicity; Fitzpatrick skin type I-III (25); dull complexion; normal body mass index; absence of current or prior skin diseases; compliance to abstain during study period from any topical application of compounds, with the exception of standard cleaning soap; and no know food intolerances.

All subjects were subjected to a 2-week washout period, during which they discontinued all moisturizer use and substituted their body cleanser with a supplied mild body wash. The subjects were not taking any vitamin/mineral supplements or medication.

The healthy non-smoker subjects were randomly recruited, divided into two groups matched based on their life-style, alcohol use, body mass index and physical activity (kcal/week), and then were assigned to a study group (active or antioxidant-control group; $\mathrm{n}=30$ each), avoiding any significant differences between the groups. In the FPP-treated group, the test product was administered as a $4.5 \mathrm{~g}$ sachet sublingually twice a day (total dose, $9.0 \mathrm{~g}$ per day), $1 \mathrm{~h}$ after breakfast and lunch, and then the subjects fasted for at least $30 \mathrm{~min}$. In the antioxidant-control group, a similar-flavored sugar was administered along with antioxidants (10 mg trans-resveratrol, $60 \mu \mathrm{g}$ selenium, $10 \mathrm{mg}$ vitamin $\mathrm{E}$ and $50 \mathrm{mg}$ vitamin $\mathrm{C}$ ) in the same manner as in the FPP-treated group. The subjects received administration for 90 days and the study was designed as double-blinded. A dietary questionnaire was used, and habitual intake of macronutrient and micronutrient was also submitted using a 7-day diet history model.

Skin moisturization. A skin capacitance instrument (Nova Dermal Phase Meter 9003; Nova Technology Corporation, Portsmouth, NH, USA) was used to measure skin moisturization. Using this corneometer, data were obtained by integrating measurements at different frequencies of the applied current at preselected frequencies up to $1 \mathrm{MHz}$. Capacitance values were calculated from the phase delay of the signal. The standard probe had two concentric brass ring electrodes separated by a 1-mm isolator, and there was direct Galvanic contact between the electrodes and the skin. The final values are provided in arbitrary units (26).

Measurements were performed at five different sites on the cheeks. The arithmetic mean for each volunteer and time point were calculated.

Skin elasticity. Facial (malar area) skin elasticity was calculated through a non-invasive suction skin elasticity device (Cutometer dual MPA 580; Courage and Khazaka Electronic $\mathrm{GmbH}$, Cologne, Germany). This device generated a graph by measuring skin extensibility (Ue), delayed distension (Uv), final deformation (Uf), immediate retraction (Ur), total recovery (Ua) and residual deformation at the end of measuring cycle (R). Using an optical measuring probe, this device derives the aforementioned measurements through the principle of suction skin-fold elongation (27). A 2-mm measuring probe applied a constant suction of 350 mbar for $18 \mathrm{sec}$ followed by a 2 -sec relaxation interval, and this was repeated twice.

Out of the measured $\mathrm{R}$ parameters retrieved by the Cutometer, the values of R2, R5 and R6 were examined. 
$\mathrm{R} 2$ represents the gross-elasticity of the skin, including the viscous deformation, and it is expressed by the ratio of skin deformation over the ultimate distension (Ua/Uf) (28). Values closer to 1 represented higher elastic properties of the skin. R5, which is calculated by immediate retraction (Ur) / skin extensibility (Ue), is associated with the net skin elasticity without the interference of viscous deformation $(5,8)$. Similarly, values close to 1 were associated with increased elastic properties of the skin. The ratio of $\mathrm{Ur} / \mathrm{Ue}$ is the most significant parameter for assessing skin aging, since it mirrors the elastic recovery, which is known to decline with aging (29), subsequent to deformation and independently from the skin thickness. By contrast, the factor R6 is the most reliable index of epidermal and dermal water content (27), representing the role of viscoelasticity and viscous deformation components against the elastic deformation of the skin due to the increased interstitial fluid through the fibrous mesh (28). R6 is represented by the ratio of viscoelasticity over skin extensibility (Uv/Ue).

Skin surface and brown spot intensity. The skin surface properties (including wrinkle depth and roughness) and the brown spot intensity were assessed using standardized digital photographs captured with the Visia-CR imaging system (Canfield Scientific, Inc., Fairfield, NJ, USA). This is a multi-spectral imaging device that uses a specialized software detecting and analyzing dark spots directly on the captured photographs (30). The analysis allows the assessment of the color intensity and the size of specific dark spots, as well as the assessment of the overall face complexion (skin evenness). Evenness in skin color and texture was identified based on gradations in skin color compared with the surrounding skin tone. The skin evenness evaluation was performed by an expert evaluator at specified study intervals. The evaluator assessed each facial parameter using a modified $100 \mathrm{~mm}$ visual analog scale (VAS) expressing the perception of unevenness, with lower scores indicating a more even and less aged skin appearance (31). The evaluator selected a location on the VAS that corresponded with the perception of the subject's skin in relation to the labelled vertical positions on the scale. The distance between the mark recorded and the left origin of the line was subsequently measured in millimeters to allow for the assignment of a numerical score for the extent and/or severity of the evaluated parameter.

Skin sampling. Following enrollment, the condition of all the subjects was initially stabilized for $30 \mathrm{~min}$, in a climate$\left(22 \pm 2^{\circ} \mathrm{C}\right)$ and humidity-controlled $(50 \pm 10 \%)$ room. Next, skin samples were collected using Corneofix ${ }^{\circledR}$ foils (Courage and Khazaka Electronic $\mathrm{GmbH}$ ), and 3-ml punch biopsies were obtained from the extensory side of the patient forearms. Samples were also collected after 90 days.

Gene expression studies. Genes recently highlighted (18-20) as playing a relevant role in aging and oxidative protection (including AQP-3, CyPA and CD147) along with an intrinsic aging-associated gene (progerin) were investigated. Reverse transcription-polymerase chain reaction (RT-PCR) was used to determine their expression at 0 days and after 90 days.

RNA extraction and RT-PCR analysis. Briefly, cells were lysed by Ambion lysis buffer (Ambion, Carlsbad, CA, USA) for
$20 \mathrm{~min}$ and the lysates were mixed with an equal volume of $64 \%$ ethanol. The lysates were transferred to a mini-column and centrifuged at $10,000 \times \mathrm{g}$ for $1 \mathrm{~min}$. The column was then washed with $700 \mu \mathrm{l}$ wash buffer 1 and twice with $500 \mu \mathrm{l}$ wash buffer 2/3 (GenePharma Co., Ltd., Shanghai, China). Following incubation with $50 \mu$ l elution buffer (GenePharma Co., Ltd.), the resulting flow was retrieved and $50 \mu \mathrm{l}$ elution buffer was added followed by centrifugation at $10,000 \mathrm{x}$. Next, $1 \mu \mathrm{l}$ DNAse I was added to $20 \mu \mathrm{l}$ of RNA solution with suitable DNAse I buffer (GenePharma Co., Ltd.) and incubated at $37^{\circ} \mathrm{C}$ for $2 \mathrm{~h}$. The DNAse I was removed by adding DNAse removing reagent and the purified RNA was retrieved by centrifugation at $10,000 \mathrm{xg}$ for $1 \mathrm{~min}$. The quantity of total RNA was first assessed by measuring the optical density at $260 \mathrm{~nm}$, and the quality of the total RNA was estimated using $1.5 \%$ agarose gel electrophoresis. RT was performed in a 20 -ml reaction solution containing $3 \mu \mathrm{g}$ total RNA using the Revert cDNA Synthesis kit (Toyobo Biotech, Co., Ltd., Shanghai China). PCR was performed in a thermal cycler with preliminary denaturation at $94^{\circ} \mathrm{C}$ for $5 \mathrm{~min}$, followed by amplification for 30 cycles of denaturation at $94^{\circ} \mathrm{C}$ for $40 \mathrm{sec}$, annealing at $65^{\circ} \mathrm{C}$ for $1 \mathrm{~min}$ and extension at $72^{\circ} \mathrm{C}$ for $1 \mathrm{~min}$. Subsequently, $5 \mu \mathrm{l}$ PCR product was separated by electrophoresis on a $1.5 \%$ agarose gel, and visualized using ethidium bromide staining under UV light. The primers used were as follows: AQP3 forward, 5'-AGACAGCCCCTTCAG GATTT-3', and reverse, 5'-TCCCTTGCCCTGAATATCTG-3'; CypA forward, 5'-GTCAACCCCACCGTGTTCTTC-3', and reverse, 5'-TTTCTGCTGTCTTTGGGACCTTG-3'; CD147 forward, 5'-TCGCGCTGCTGGGCACC-3', and reverse, 5'-TGGCGCTGTCATTCAAGGA-3'; progerin forward, 5'-ACTGCAGCAGCTCGGGG-3', and reverse, 5'-GGCTCT GGGCTCCTGAGCC-3'; and glyceraldehyde-3-phosphate dehydrogenase (GAPDH) forward, 5'-ACCACAGTCCATGCC ATCAC-3', and reverse, 5'-TCCACCACCCTGTTGCTGTA-3'. The PCR products were subjected to electrophoresis on a $1.4 \%$ agarose gel using a Gel Doc 2000 fluorescence documentation system (Bio-Rad Laboratories Co., Ltd., Shanghai, China) and GeneScan analysis software (version 672; Applied Biosystems; Thermo Fisher Scientific, Inc., Waltham, MA, USA). The mRNA level of each sample was normalized to that of the $\beta$-actin mRNA.

Dermal redox balance and nitrogen oxide (NO) assessment. After the patients fasted overnight, the antioxidant capacity of the skin was assessed on the third and fourth skin strips (Corneofix ${ }^{\circledR}$ foils were used after discarding the first and second layers).

Determination of lipid peroxidation. The lipid peroxidation product was determined by measuring the malondialdehyde (MDA) content in tissue homogenates. Skin samples were immediately frozen to $-70^{\circ} \mathrm{C}$ until assayed for thiobarbituric acid reactive species (TBARS) formation. As an index of oxidative stress, the formation of TBARS during an acid-heating reaction was used. Briefly, the samples were mixed with $1 \mathrm{ml}$ trichloroacetic acid (10\%) and $1 \mathrm{ml}$ thiobarbituric acid $(0.67 \%)$ and then heated in a boiling water bath for $15 \mathrm{~min}$. The TBARS content was determined by measuring the absorbance at $535 \mathrm{~nm}$, using 1,1,3,3-tetramethoxypropane as an external standard. The results are expressed as malondialdehyde 
equivalents per milligram of protein (Lowry assay), and values are expressed in units of $\mathrm{nM} / \mathrm{mg}$ protein.

Superoxide dismutase (SOD) activity measurement. SOD production was evaluated using a lucigenin-amplified chemiluminescence method. Skin samples were placed in Krebs buffer (Sigma-Aldrich, St. Louis, MO, USA) at pH 7.40, for $20 \mathrm{~min}$. The samples were then transferred to a counting vial under light protection and immersed in a 5- $\mu \mathrm{M}$ lucigenin solution (Sigma-Aldrich) in Krebs buffer (total volume, $1.0 \mathrm{ml}$ ) mimicking tissue generation of superoxide. The emitted luminescence was calculated for $5 \mathrm{~min}$ in a Berthold Biolumat luminometer (Berthold Detection Systems GmbH, Pforzheim, Germany). Background signals from the buffer and lucigenin were subtracted from the tissue sample signals and the final value was normalized for dry weight. Chemiluminescence was measured directly and continuously for $5 \mathrm{~min}$ to obtain basal values. Data are expressed as counts per min per mg of dry tissue.

In vivo NO assessment. Following a thorough skin cleansing, a sterilized microdialysis probe was gently inserted into the forearm skin while carefully avoiding fluid exudation. Next, $0.3 \mathrm{ml}$ of $100 \mu \mathrm{M} 4,4$ '-sulfonyldianiline in sterile normal saline (pH 5.8-6.0) was fed to a microdialysis tube and maintained for 20 min to allow for local absorption of NO. The total $\mathrm{NO}_{\mathrm{x}}$ concentration $\left(\mathrm{NO}_{2}{ }^{-}\right.$and $\left.\mathrm{NO}_{3}{ }^{-}\right)$was calculated in the dialysate using the ozone phase chemiluminescence method (NOA280i; GE Analytical Instruments, Boulder, CO, USA), with a lowest sensitivity of $1.0 \mathrm{pM}$. A $5 \mu \mathrm{l}$ sample of fresh dialysate was then reduced by a vanadium(III)/HCl solution. The resulting $\mathrm{NO}$ gas was reacted with ozone to generate a chemiluminescent reaction, and the nitrate calibration curve was set using graded concentrations of $\mathrm{NaNO}_{3}$ diluted in sterile nitrogen-free water. The whole quantity of $\mathrm{NO}_{3}$ in each dialysate sample was measured by correlating the signal peaks through nitrate curve calibration. Triplicate measurements were performed. The presence of $\left(\mathrm{NO}_{2}^{-}\right)$in the dialysate samples was equalled to the water basal level so that the final $\mathrm{NO}_{\mathrm{x}}$ concentration in the dialysate was expressed in $\mu \mathrm{M}$ units (32).

Intraobserver and interobserver variability. Intraobserver and interobserver variability for clinical testing were estimated by calculating the mean and $95 \%$ confidence interval (CI) of the arithmetic differences among three consecutive measurements obtained by a single researcher on the same volunteer. Variability was expressed the mean \pm 1.96 (which was the standard deviation value of the mean arithmetic difference), according to Bland and Altman (33). When the differences were normally distributed, $95 \%$ of the differences were within a range of $\mathrm{SD}$ of the mean difference $=1.96$.

Statistical analyses. Statistical analyses were performed using Prism 5 software (GraphPad Software, Inc., San Diego, CA, USA). Data are expressed as the mean of three independent experiments, and were analyzed by Student's t-test and analysis of variance (ANOVA). In addition, ANOVA with Dunnett's test was used to compare VAS scores for clinical grading of facial skin attributes or, more specifically, to compare differences between the values at baseline (day 0) and after 90 days of

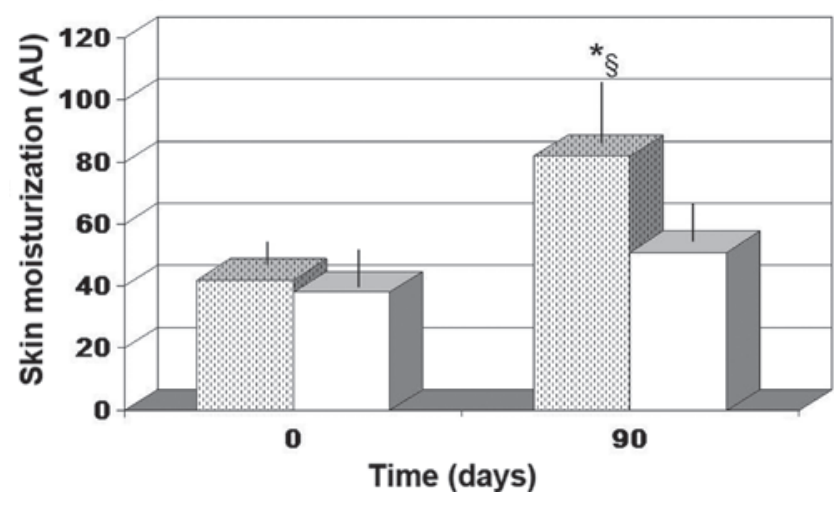

Figure 1. Skin moisturization. ${ }^{*} \mathrm{P}<0.05$ vs. baseline value and ${ }^{\S} \mathrm{P}<0.05$ vs. antioxidant-control. Dotted columns, treated with FPP; white columns, treated with an antioxidant-control; FPP, fermented papaya preparation.

observation. Genes expression level comparisons were tested by Kruskal-Wallis t-test, and the correlation analysis was assessed with Spearman's rho method. $\mathrm{P}<0.05$ was considered to indicate a statistically significant difference.

\section{Results}

Skin moisturization. Antioxidant cocktail supplementation was not found to affect skin moisturization. By contrast, FPP supplementation resulted in a significant improvement in skin moisturization after 90 days ( $\sim 95 \%$ increase; $\mathrm{P}<0.04$; Fig. 1$)$.

Skin elasticity. As compared to the baseline value, the FPP-treated group showed a non-significant increase in the $\mathrm{R} 2$ values at 90 days of observation $(\mathrm{P}<0.063$; Fig. 2A). By contrast, the values obtained from the antioxidant-treated group at 90 days were unchanged compared with the baseline control values. Notably, the control values detected after 90 days of observation for R6 (Uv/Ue) were significantly lower compared with those obtained in the FPP-treated group $(\mathrm{P}<0.05)$. For factor R5, no significant changes occurred, regardless of the treatment employed (Fig. 2B). However, the FPP-treated group showed a non-significant increase at 90 days compared with the baseline value $(\mathrm{P}>0.05)$. Furthermore, FPP supplementation maintained for 90 days resulted in a statistically significant increase in the R6 values when compared with the baseline value $(\mathrm{P}<0.01)$ and with the antioxidant-control group $(\mathrm{P}<0.05$; Fig. 2C).

Skin surface and brown spot intensity. The majority of the parameters tested, including roughness, wrinkle depth and darkness of brown spots, did not show a statistical significant change for the two treatment employed (data not shown). However, the overall evenness (color variation in the skin tone) was evaluated as significantly improved in the FPP-treated group $(\mathrm{P}<0.05)$, but not in the antioxidant-control group (Fig. 3).

Skin gene expression experiments. Gene expression experiments demonstrated that, as compared with the baseline and 90-day values observed in the antioxidant-control group, AQP3 expression was significantly upregulated after 90 days of FPP 

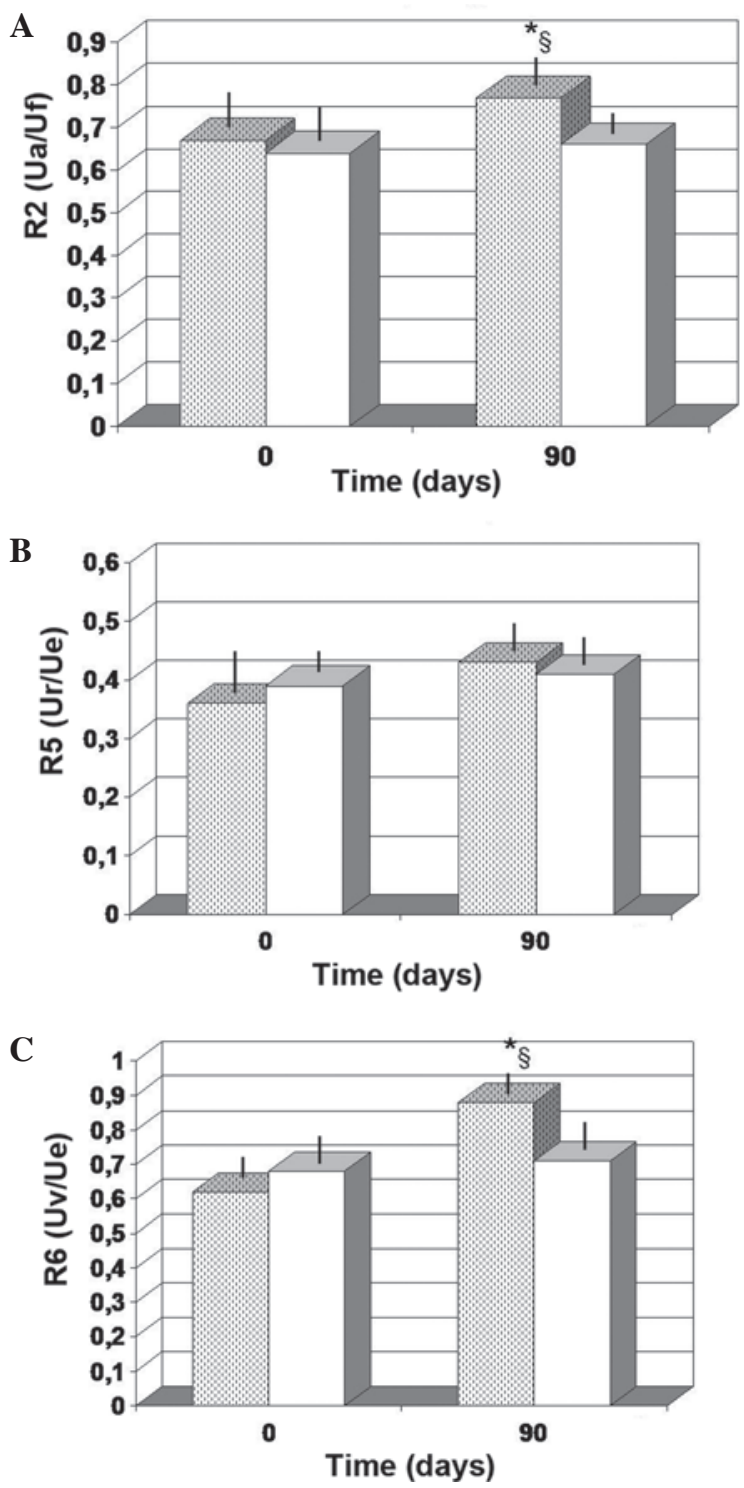

Figure 2. Skin elasticity parameters. (A) R2 (Ua/Uf); (B) R5 (Ur/Ue); (C) R6 (Uv/Ue). ${ }^{*} \mathrm{P}<0.01$ vs. baseline, and ${ }^{\S} \mathrm{P}<0.05$ vs. antioxidant-control. Dotted columns, treated with FPP; white columns, treated with an antioxidant-control; FPP, fermented papaya preparation.

administration, whereas CyPA and CD147 expression levels were significantly downregulated $(\mathrm{P}<0.05$; Figs. 4 and 5$)$. By contrast, the antioxidant-supplemented group did not show a significant downregulation in the levels of CD147, after 90 days of treatment ( $\mathrm{P}>0.05$ vs. baseline value). Progerin gene expression was unaffected by the two treatments, although a trend towards decreased expression was noted in the FPP-supplemented group $(\mathrm{P}<0.068$, non-significant change; Fig. 5).

Dermal redox balance and NO assessment. The two treatments were found to significantly decrease the MDA level in the tissue, as compared with the baseline values $(\mathrm{P}<0.05)$. In addition, there was no significant difference between the two different treatment groups (Fig. 6A). A significant increase in the SOD level was also noted after 90 days $(\mathrm{P}<0.01$ vs. baseline values). However, FPP treatment enabled a more significant increase in the SOD tissue concentration $(\mathrm{P}<0.05$ vs.

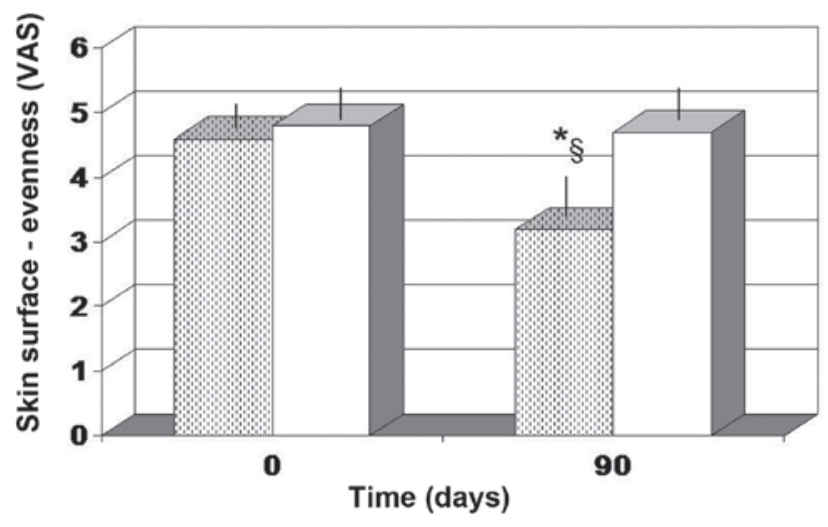

Figure 3. Skin surface evenness, determined based on the VAS score. The score was interpreted as a perception of unevenness, thus a lower score indicated higher evenness. ${ }^{*} \mathrm{P}<0.05$ vs. baseline and ${ }^{\S} \mathrm{P}<0.05$ vs. antioxidant-control. Dotted columns, treated with FPP; white columns, treated with an antioxidant-control; VAS, visual analog scale; FPP, fermented papaya preparation.

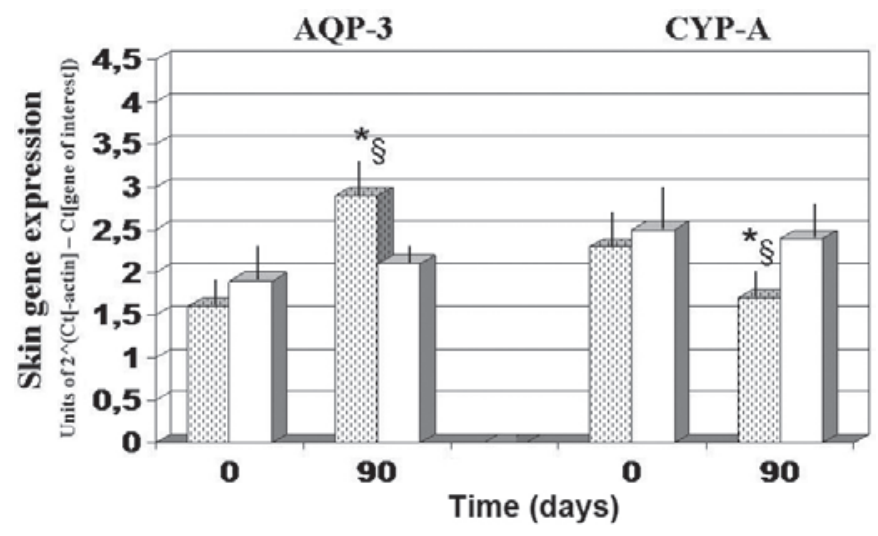

Figure 4. Skin gene expression of AQP-3 and CyPA. ${ }^{*} \mathrm{P}<0.05$ vs. baseline and ${ }^{\S} \mathrm{P}<0.05$ vs. antioxidant-control. Dotted columns, treated with FPP; white columns, treated with an antioxidant-control; FPP, fermented papaya preparation; AQP-3, aquaporin-3; .CyPA, cyclophilin A.

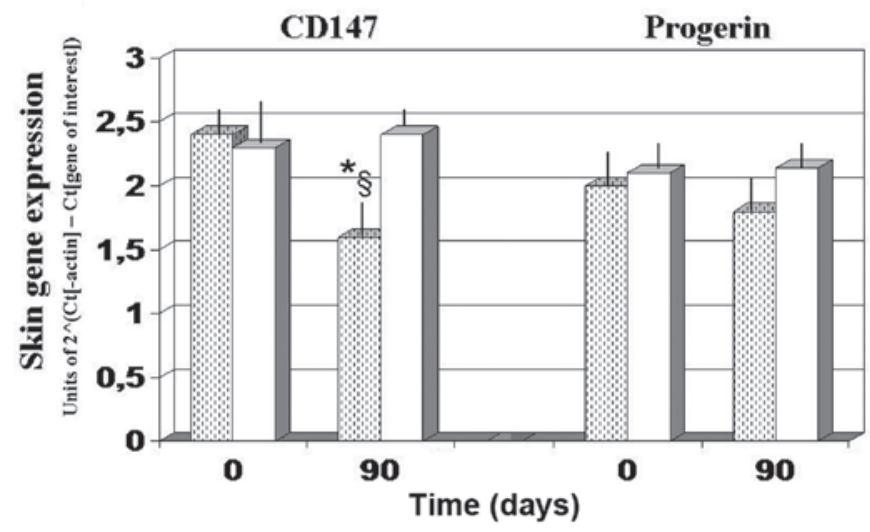

Figure 5. Skin gene expression of CD147 and progerin. ${ }^{*} \mathrm{P}<0.05$ vs. baseline and ${ }^{\S} \mathrm{P}<0.05$ vs. antioxidant-control. Dotted columns, treated with FPP; white columns, treated with antioxidant-control; FPP, fermented papaya preparation.

antioxidant-control group; Fig. 6B). In addition, only FPP showed a statistically significant increase in local NO production $(\mathrm{P}<0.05$ vs. baseline value and vs. antioxidant-control group; Fig. 7). 
A

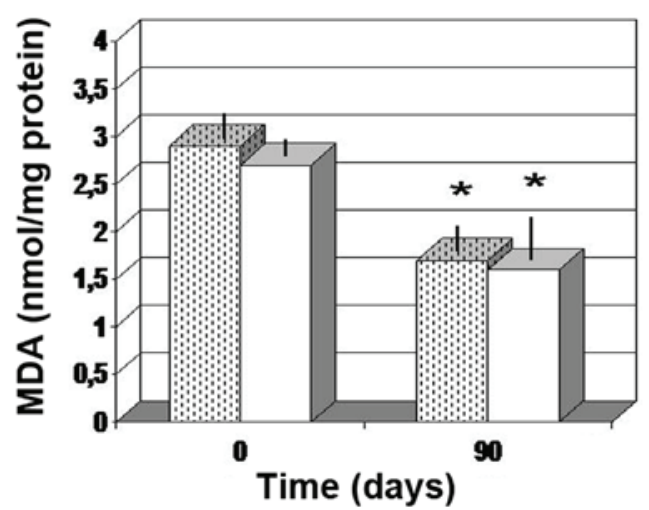

B

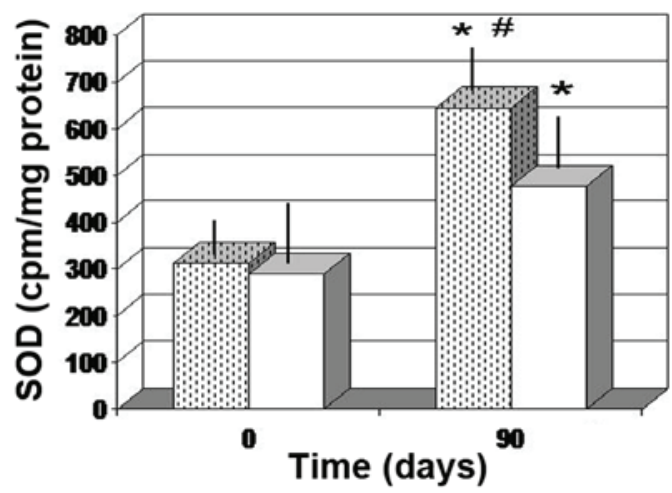

Figure 6. Skin redox balance of (A) MDA and (B) SOD. ${ }^{*} \mathrm{P}<0.05$ vs. baseline; ${ }^{\#} \mathrm{P}<0.05$ vs. antioxidant-control and $\mathrm{P}<0.01$ vs. baseline. Dotted columns, treated with FPP; white columns, treated with an antioxidant-control; FPP, fermented papaya preparation; MDA, malondialdehyde; SOD, superoxide dismutase.

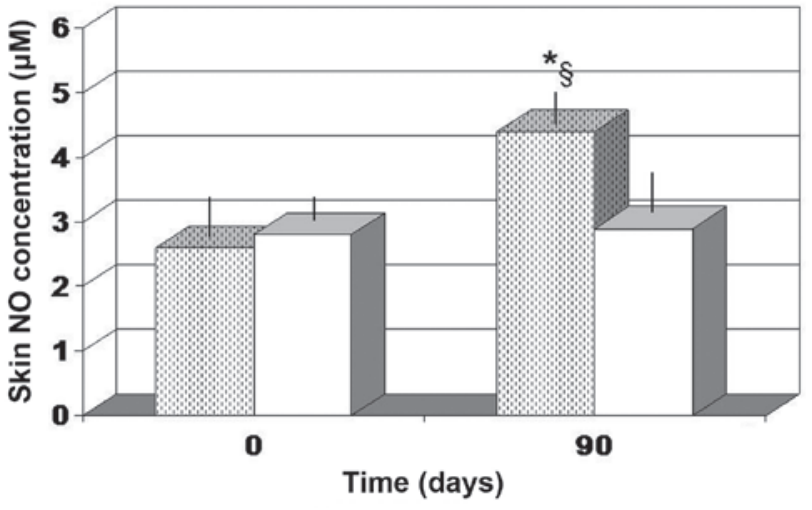

Figure 7. Skin NO concentration. ${ }^{*} \mathrm{P}<0.05$ vs. baseline and ${ }^{\S} \mathrm{P}<0.05$ vs. antioxidant-control. Dotted columns, treated with FPP; white columns, treated with an antioxidant-control; FPP, fermented papaya preparation.

\section{Discussion}

Aging is a complex process that underlies multiple factors, with the involvement of heritable and several environmental effects. In particular, aging is influenced by large-scale epigenomic expression changes; however, the ways of modulating such functional variations in order to achieve health benefits in humans require further investigation. Skin cells are constantly exposed to ROS and oxidative stress from exogenous and endogenous sources (34-36). In addition, the skin is naturally endowed with a variety of low molecular weight antioxidants and antioxidant enzymes with ROS-scavenging activity, forming an elaborate inducible/adaptive redox system (37). This system consists of enzymatic antioxidants, including SOD, glutathione peroxidase and catalase, as well as of non-enzymatic antioxidants, such as vitamin $\mathrm{C}$, vitamin $\mathrm{E}$ isoforms, ubiquinol, glutathione and uric acid. These antioxidants act together with other factors, such as ascorbate, carotenoids and sulfhydrils, to regulate the redox system. However, this antioxidant machinery is not fully efficient; this becomes more evident during the process of aging, in which an uncontrolled oxidative damage to the proinflammatory cytokines occurs, which is also the basis of carcinogenesis transformation (38). Certain abnormalities observed in photoaging can be also found in chronoaging, and these include the loss of dermal collagen that is observed at a lesser extent in chronoaging. However, this also suggests that tissue injuries initially triggered by environmental factors, such as sun exposure, may indeed accelerate intrinsic aging mechanisms and help to accumulate oxidized/degraded proteins (39).

From a macroscopic viewpoint, the present study demonstrated that, unlike the antioxidant-control treatment, the FPP nutraceutical resulted in a significant improvement in the skin moisturization and elasticity, and namely in its viscous elastic component. This finding is notable and can be tentatively linked to the significant upregulation of AQP-3 expression that was exerted only by this specific fermented nutraceutical. To add more relevance to these findings, it is worth mentioning that Cao et al (40) have demonstrated that UV may induce AQP3 downregulation in human skin keratinocytes and this is also likely to occur in vivo. Notably, a recent study (41) suggested that resveratrol treatment downregulated the expression of AQP-3, at least in specific experimental set-ups, indicating that this treatment may be more suitable for hyperplastic skin disorders, rather than for the overall skin health.

Although the two nutraceuticals used in the current study did not show any overt effect on the wrinkle and age spots intensity, it was interesting to observe that FPP resulted in a significant improvement in skin complexion (based on the skin evenness, as evaluated by an expert investigator who was blinded to the treatment). This clinical finding may be associated with the obtained data of higher hydrated derma and possibly also due to the higher concentration of NO. As a matter of fact, this unique NO-modulating effect of FPP was demonstrated in an experimental model established by Collard and Roy (42), while a recent study by our group investigated the cardiovascular benefits of FPP in healthy subjects (43). The two treatments seen in the present study enabled a significant improvement of the redox balance, and this may help counteract the increased MDA and decreased SOD levels that were observed in skin injury caused by UV and intrinsic aging (39). However, unlike the antioxidant-control treatment, FPP exerted a significant downregulating action on the gene expression levels of CyPA and CD147. CyPA has been previously shown to be overexpressed in several cancer cell lines (44). In addition, Han et al (17) recently provided convincing data in support of the oncogenic role of CyPA in skin tumorigenesis, including squamous and basal cell carcinomas, which are essentially caused by UV damage. The transmembrane glycoprotein CD147 has been found to play a similar role, since it is strictly 
connected to CyPA $(45,46)$, and therefore the CyPA-CD147 complex is suggested to be a novel therapeutic target (47). Thus, the aforementioned data provide further evidence that a systemic redox-modulating approach (with FPP supplementation in the case of the present study) is certainly noteworthy. In recent years, a number of studies have investigated the brain-skin connection $(48,49)$, providing new insights from the understanding and interventional perspective viewpoints. Furthermore, a recent study reported that CyPA/CD147 modulation via the extracellular regulated protein kinase signaling pathway may play a relevant role in the brain physiopathology of dementia (50). In fact, at a neurobiology center in Italy, a study has demonstrated a significant improvement in Parkinson patients using FPP, both biochemically and clinically (51). In particular, among several parameters, a significant decrease was observed in 8-hydroxy-2'-deoxyguanosine, which is know to increase also in the skin of Parkinson patients (52).

Progerin can also be induced by UV, and its accumulation is known to be involved in the process of DNA repair and signaling during cellular injury (53). In the present study, none of the supplemental interventions affected the progerin gene expression. It is hypothesized that this fundamental biological parameter may require a longer treatment period in order to show a response, but also that oxidative stress-associated factors are only part of a larger epigenomic environment to be advocated for its modulation.

Although investigations involving dietary and frequently high intake of a single antioxidant or vitamin may be highly flawed due to the interactive nature of antioxidants in vivo, large-scale nutrition and nutraceutical intervention studies can aid the validation of nutrigenomic-dermagenomic strategies for specific genes within an overall integrative health medical approach. Nonetheless, the findings of the present study findings have demonstrated that FPP possesses a distinct profile among food supplements and functional foods by effectively acting at a biochemical/epigenomic skin level. Furthermore, the importance of well-designed topical formulations for skin protection maintains its utmost importance and opens new avenues of treatment $(54,55)$.

In conclusion, based on the present findings, orally administered FPP appears to result in a consistent biological and gene regulatory improvement in the skin, while common oral antioxidants had only a minor effect.

\section{References}

1. Jones DP: Redox theory of aging. Redox Biol 5: 71-79, 2015.

2. Kammeyer A and Luiten RM: Oxidation events and skin aging. Ageing Res Rev 1: 16-29, 2015.

3. Choi CM and Berson DS: Cosmeceuticals. Semin Cutan Med Surg 25: 163-168 2006.

4. Katiyar SK: Green tea prevents non-melanoma skin cancer by enhancing DNA repair. Arch Biochem Biophys 508: 152-158, 2011.

5. Udompataikul M, Sripiroj P and Palungwachira P: An oral nutra-ceutical containing antioxidants, minerals and glycosaminoglycans improves skin roughness and fine wrinkles. Int J Cosmet Sci 31: 427-435, 2009.

6. Izumi T, Saito M, Obata A, Arii M, Yamaguchi H and Matsuyama A: Oral intake of soy isoflavone aglycone improves the aged skin in adult women. J Nutr Sci Vitaminol (Tokyo) 53: 57-62, 2007.

7. Natarajan VT, Ganju P, Ramkumar A, Grover R and Gokhale R: Multifaceted pathways protect human skin from UV radiation. Nat Chem Biol 10: 542-551, 2014.
8. Uitto J: The role of elastin and collagen in cutaneous aging: Intrinsic aging versus photoexposure. J Drugs Dermatol 7 (Suppl 2): S12-S6, 2008

9. Rabe JH, Mamelak AJ, McElgunn PJ, Morison WL and Sauder DN: Photoaging: Mechanisms and repair. J Am Acad Dermatol 55: 1-19, 2006.

10. Yaar M and Gilchrest BA: Photoageing: mechanism, prevention and therapy. Br J Dermatol 157: 874-887, 2007.

11. Melnikova VO and Ananthaswamy HN: Cellular and molecular events leading to the development of skin cancer. Mutat Res 571: 91-106, 2005.

12. Makrantonaki E and Zouboulis CC; German National Genome Research Network 2: The skin as a mirror of the aging process in the human organism-state of the art and results of the aging research in the German National Genome Research Network 2 (NGFN-2). Exp Gerontol 42: 879-886, 2007.

13. Makrantonaki E, Schonknecht P, Hossini AM, Kaiser E, Katsouli MM, Adjaye J, Schröder J and Zouboulis CC: Skin and brain age together: The role of hormones in the ageing process. Exp Gerontol 45: 801-813, 2010.

14. Buonocore D, Lazzeretti A, Tocabens P, Nobile V, Cestone E, Santin G, Bottone MG and Marzatico F: Resveratrol-procyanidin blend: Nutraceutical and antiaging efficacy evaluated in a placebo-controlled, double-blind study. Clin Cosmet Investig Dermatol 5: 159-165, 2012.

15. Gruber JV and Holtz R: Examining the genomic influence of skin antioxidants in vitro. Mediators Inflamm 2010: 230450, 2010.

16. Hara-Chikuma M and Verkman AS: Aquaporin-3 facilitates epidermal cell migration and proliferation during wound healing. J Mol Med (Berl) 86: 221-231, 2008.

17. Han W, Soltani K, Ming M and He YY: Deregulation of XPC and CypA by cyclosporin A: An immunosuppression-independent mechanism of skin carcinogenesis. Cancer Prev Res (Phila) 5: 1155-1162, 2012.

18. Satoh K, Satoh T, Kikuchi N, Omura J, Kurosawa R, Suzuki K, Sugimura K, Aoki T, Nochioka K, Tatebe S, et al: Basigin mediates pulmonary hypertension by promoting inflammation and vascular smooth muscle cell proliferation. Circ Res 115: 738-750, 2014.

19. Ramachandran S, Venugopal A, Kutty VR, A V, GD, Chitrasree V, Mullassari A, Pratapchandran NS, Santosh KR, Pillai MR and Kartha CC: Plasma level of cyclophilin A is increased in patients with type 2 diabetes mellitus and suggests presence of vascular disease. Cardiovasc Diabetol 13: 38, 2014.

20. Iacono KT, Brown AL, Greene MI and Saouaf SJ: CD147 immunoglobulin superfamily receptor function and role in pathology. Exp Mol Pathol 83: 283-295, 2007.

21. Takeuchi H and Rünger TM: Longwave UV light induces the aging-associated progerin. J Invest Dermatol 133: 1857-1862, 2013 .

22. Marotta F, Yoshida C, Barreto R, Naito Y and Packer L: Oxidative-inflammatory damage in cirrhosis: Effect of vitamin $\mathrm{E}$ and a fermented papaya preparation. J Gastroenterol Hepatol 22: 697-703, 2007.

23. Aruoma OI, Colognato R, Fontana I, Gartlon J, Migliore L, Koike K, Coecke S, Lamy E, Mersch-Sundermann V, Laurenza I, et al: Molecular effects of fermented papaya preparation on oxidative damage, MAP Kinase activation and modulation of the benzo[a]pyrene mediated genotoxicity. Biofactors 26: 147-159, 2006.

24. Bertuccelli G, Marotta F, Zerbinati N, Cabeca A, He F, Jain S, Lorenzetti A, Yadav H, Milazzo M, Calabrese F, et al: Iron supplementation in young iron-deficient females causes gastrointestinal redox imbalance: Protective effect of a fermented nutraceutical. J Biol Regul Homeost Agents 28: 53-63, 2014.

25. Sachdeva S: Fitzpatrick skin typing: Applications in dermatology. Indian J Dermatol Venereol Leprol 75: 93-96, 2009.

26. Clarys P, Barel AO and Gabard B: Non-invasive electrical measurements for the evaluation of the hydration state of the skin: Comparison between three conventional instruments - the Corneometer, the Skicon, and the Nova DPM. Skin Res Technol 5: 14-20, 1999.

27. Courage W: Hardware and measuring principle: corneometer. In: Bioengineering of the Skin: Water and the Stratum Corneum. Elsner P, Berardesca E and Maibach HI (eds). CRC Press, Boca Raton, FL, 1994.

28. Barel AO, Courage W and Clarys P: Suction method for measurement of skin mechanical properties: The cutometer. In: Handbook of Non-invasive methods and the Skin. Serup J and Jemec GBE (eds). CRC Press, Boca Raton, FL, 1995. 
29. Han A, Chien AL and Kang S: Photoaging. Dermatol Clin 32: 291-299, 2014.

30. Wilhelm KP, Elsner P, Berardesca E and Maibach HI (eds): Bioengineering of the skin: Skin surface imaging and analysis. CRC Press, Boca Raton, FL, 1996.

31. Luebberding S, Krueger N and Kerscher M: Comparison of Validated Assessment Scales and 3D digital fringe projection method to assess lifetime development of wrinkles in men. Skin Res Technol 20: 30-36, 2014.

32. Ding TM, Chen J, Zhang ZX and Zhang YH: The methods for determination of nitric oxide in vivo and their applications. Yao Xue Jin Zhan 29: 221-226, 2005 (In Chinese).

33. Bland JM and Altman DG: Applying the right statistics: Analyses of measurement studies. Ultrasound Obstet Gynecol 22: 85-93, 2003.

34. Indo HP, Yen HC, Nakanishi I, Matsumoto K, Tamura M, Nagano Y, Matsui H, Gusev O, Cornette R, Okuda T, et al: A mitochondrial superoxide theory for oxidative stress diseases and aging. J Clin Biochem Nutr 56: 1-7, 2015.

35. Vajapey R, Rini D, Walston J, Abadir P. The impact of age-related dysregulation of the angiotensin system on mitochondrial redox balance. Front Physiol 5: 439, 2014.

36. Fulop T, Witkowski JM, Pawelec G, Alan C, Larbi A. On the immunological theory of aging. Interdiscip Top Gerontol 39: 163-176, 2014

37. Poljšak B, Dahmane RG and Godić A: Intrinsic skin aging: The role of oxidative stress. Acta Dermatovenerol Alp Pannonica Adriat 21: 33-36, 2012.

38. Godic A, Poljšak B, Adamic M and Dahmane R: The role of antioxidants in skin cancer prevention and treatment. Oxid Med Cell Longev 2014: 860479, 2014.

39. Lu CY, Lee HC, Fahn HJ and Wei YH: Oxidative damage elicited by imbalance of free radical scavenging enzymes is associated with large-scale mtDNA deletions in aging human skin. Mutat Res 423: 11-21, 1999.

40. Cao C, Wan S, Jiang Q, Amaral A, Lu S, Hu G, Bi Z, Kouttab N, Chu W and Wan Y: All-trans retinoic acid attenuates ultraviolet radiation-induced down-regulation of aquaporin-3 and water permeability in human keratinocytes. J Cell Physiol 215: 506-516, 2008.

41. Wu Z, Uchi H, Morino-Koga S, Shi W and Furue M Resveratrol inhibition of human keratinocyte proliferation via SIRT1/ARNT/ERK dependent downregulation of aquaporin 3. J Dermatol Sci 75: 16-23, 2014.

42. Collard E and Roy S: Improved function of diabetic wound-site macrophages and accelerated wound closure in response to oral supplementation of a fermented papaya preparation. Antioxid Redox Signal 13: 599-606, 2010.

43. Marotta F, Yadav H, Kumari A, Catanzaro R, Jain S, Polimeni A, Lorenzetti A and Soresi V: Cardioprotective effect of a biofermented nutraceutical on endothelial function in healthy middle-aged subjects. Rejuvenation Res 15: 178-181, 2012.
44. Lee J and Kim SS: An overview of cyclophilins in human cancers. J Int Med Res 38: 1561-1574, 2010.

45. Ayva SK, Karabulut AA, Akatli AN, Atasoy P and Bozdogan O: Epithelial expression of extracellular matrix metalloproteinase inducer/CD147 and matrix metalloproteinase-2 in neoplasms and precursor lesions derived from cutaneous squamous cells: An immunohistochemical study. Pathol Res Pract 209: 627-634, 2013.

46. Wang CH, Rong MY, Wang L, Ren Z, Chen LN, Jia JF, Li XY, Wu ZB, Chen ZN and Zhu P: CD147 up-regulates calcium-induced chemotaxis, adhesion ability and invasiveness of human neutrophils via a TRPM-7-mediated mechanism. Rheumatology (Oxford) 53: 2288-2296, 2014.

47. Yurchenko V, Constant S, Eisenmesser E and Bukrinsky M: Cyclophilin-CD147 interactions: A new target for anti-inflammatory therapeutics. Clin Exp Immunol 160: 305-317, 2010.

48. Chen Y and Lyga J: Brain-skin connection: Stress, inflammation and skin aging. Inflamm Allergy Drug Targets 13: 177-190, 2014.

49. Doppler K, Ebert S, Uçeyler N, Trenkwalder C, Ebentheuer J, Volkmann J and Sommer C: Cutaneous neuropathy in Parkinson's disease: A window into brain pathology. Acta Neuropathol 128: 99-109, 2014.

50. Kanyenda LJ, Verdile G, Martins R, Meloni BP, Chieng J, Mastaglia F, Laws SM, Anderton RS and Boulos S: Is cholesterol and amyloid- $\beta$ stress induced CD147 expression a protective response? Evidence that extracellular cyclophilin a mediated neuroprotection is reliant on CD147. J Alzheimers Dis 39: 545-556, 2014

51. Mantello M, Catanzaro R, He F, Bissi L, Milazzo M, Lorenzetti A and Marotta F: Novel nutrigenomics avenues in nutraceuticals use: The current status of fermented papaya preparation. In: Bioactive Compounds: At the Frontier Between Nutrition and Parmacology. Aguilar Villas MV and Otero C (eds). Bentham Science, 2015 (in press).

52. del Hoyo P, García-Redondo A, de Bustos F, Molina JA, Sayed Y, Alonso-Navarro H, Caballero L, Arenas J, Agúndez JA and Jiménez-Jiménez FJ: Oxidative stress in skin fibroblasts cultures from patients with Parkinson's disease. BMC Neurol 10: 95, 2010.

53. Musich PR and Zou Y: Genomic instability and DNA damage responses in progeria arising from defective maturation of prelamin A. Aging (Albany NY) 1: 28-37, 2009.

54. Martins RM, Siqueira S, Fonseca MJ and Freitas LA: Skin penetration and photoprotection of topical formulations containing benzophenone-3 solid lipid microparticles prepared by the solvent-free spray-congealing technique. J Microencapsul 31: 644-653, 2014.

55. Scalia S, Marchetti N and Bianchi A: Comparative evaluation of different co-antioxidants on the photochemical- and functional-stability of epigallocatechin-3-gallate in topical creams exposed to simulated sunlight. Molecules 18: 574-587, 2013. 